10. Das N, Gupta R, Gupta S, et al. A real-world perspective of CD123 expression in acute leukaemia as promising biomarker to predict treatment outcome in B-ALL and AML. Clin Lymphoma Myeloma Leuk 2020; 20: e673-84.

11. Bras AE, Haas V, Stigt A, et al. CD123 expression levels in 846 acute leukemia patients based on standardised immunophenotyping. Cytometry B Clin Cytom 2019; 96B: 134-42.

12. Jiang G, Atenafu EG, Capo-Chichi JM, et al. Prognostic relevance of CD123 expression in adult AML with normal karyotype. Br J Haemato 2020; 188: $181-4$

DOI: https://doi.org/10.1016/j.pathol.2020.10.022

\section{Abnormal coagulation profiles in monoclonal gammopathy of undetermined significance: a case series}

Sir,

Monoclonal gammopathy of undetermined significance (MGUS) is a plasma cell disorder characterised by a serum monoclonal protein of less than $30 \mathrm{~g} / \mathrm{L}$, clonal bone marrow plasma cells (or lymphoplasmacytic infiltration) of less than $10 \%$ and no evidence of end organ damage. ${ }^{1}$ Plasma cell dyscrasias can be complicated by disordered haemostasis; in particular an increased risk of thrombosis is seen. ${ }^{2}$ However, a bleeding diathesis has also been reported in some patients, and abnormal coagulation study results are seen relatively commonly in patients with multiple myeloma. ${ }^{3-5}$ We report three patients referred for investigation of a prolonged activated partial thromboplastin time (APTT) who were identified to have an underlying MGUS.

The first patient was a 69 -year-old male who was referred for assessment with an incidentally detected APTT of 45 [normal range (NR) 25-35 s] and PT of 13 (NR 11-15 s) at anaesthetic pre-admission clinic. This patient had been diagnosed with a caecal tumour and had a planned robotic right hemicolectomy. He had previous surgical procedures including basal cell carcinoma removal, metatarsal shave procedure and tonsillectomy and adenoidectomy as a child, none of which were complicated by bleeding. He reported some spontaneous protracted nosebleeds approximately 20 years earlier, but no other significant bleeding. His International Society of Thrombosis and Haemostasis-Bleeding Assessment Tool (ISTH-BAT) was 1 (normal range <4). He was not receiving any antiplatelet or anticoagulant therapy.

Further investigation demonstrated that his APTT did not correct to the normal range on mixing (36 s). Specific factor level testing demonstrated a Factor XI concentration of $15 \%$ at 1:10 dilution (Table 1). With serial dilutions of plasma, all factor levels increased, suggesting possible inhibition of all factors assessed; however, this was most marked with Factor XI, which increased to a level of $60 \%$ with a 1:200 dilution (Table 1). A specific Factor XI inhibitor could not be demonstrated on Bethesda assay including a $2 \mathrm{~h}$ incubation. A lupus inhibitor was not detected. Platelet function assay100 (PFA-100) testing on this patient was normal.

Serum electrophoresis and immunofixation were performed and demonstrated an IgG kappa paraprotein measuring $3 \mathrm{~g} / \mathrm{L}$. Serum free light chain assessment demonstrated a kappa free light chain of $182 \mathrm{~g} / \mathrm{L}$ and lambda of
$14.92 \mathrm{~g} / \mathrm{L}$ (ratio 14.92). Flow cytometry of the peripheral blood demonstrated an abnormal B-cell population with a clone size of $0.3 \times 10^{9} / \mathrm{L}$ that was positive for CD19, CD20 (moderate-bright intensity), kappa (dim-moderate intensity), CD22 (dim), CD200, and negative for CD5, CD10, CD11c, CD23. There was no renal impairment, hypercalcaemia, anaemia or bony disease on a skeletal survey. Bone marrow biopsy had not been performed as we were awaiting completion of his adjuvant chemotherapy.

The factor assay findings were suspected to be secondary to interference by the $\mathrm{M}$ protein. He proceeded to his robotic right hemicolectomy with four units of fresh frozen plasma (FFP) thawed in case of bleeding. The operation proceeded without complication and FFP administration was not required. His APTT remained prolonged after resection of the caecal tumour and six cycles of adjuvant chemotherapy. His Factor XI remained low (24\% at 1:10 dilution), and all factor levels increased with serial dilution of plasma (Table 1). von Willebrand factor (VWF) studies demonstrated a VWF antigen of $172 \mathrm{U} / \mathrm{dL}$ (NR 50-200), VWF ristocetin cofactor of $188 \mathrm{U} / \mathrm{dL}$ (NR 45-200) and VWF collagen of $>200 \mathrm{U} / \mathrm{dL}$ (NR 50-200). Of note, on follow-up this patient now has two detectable IgM kappa paraproteins in addition to his IgG kappa paraprotein.

The second patient was a 70-year-old female who was referred for assessment of a prolonged APTT of $51 \mathrm{~s}$ which did not correct on mixing ( $49 \mathrm{~s}$ ) on a background of a known MGUS. She presented for elective insertion of a permanent pacemaker. She had no spontaneous bruising or bleeding but reported previous prolonged, delayed and moderately severe bleeding post-surgical procedures including prolonged bleeding following tooth extractions requiring hospital admission, post-partum haemorrhage and post-operative bleeding following dilatation and curettage, laparoscopic cholecystectomy and hysterectomy. She was not receiving any antiplatelet or anticoagulant therapy.

Intrinsic Factor assessment at 1/10 dilution demonstrated a Factor VIII of $3 \%$, Factor IX of $1 \%$, Factor XI of $1 \%$ and Factor XII of $4 \%$ using one stage factor assays (Table 1 ). These demonstrated some improvement with plasma dilution. A two stage chromogenic Factor VIII assay demonstrated a much higher level of $114 \%$ (Table 1). A specific factor inhibitor could not be identified on Bethesda assay. VWF studies were normal as were platelet count and platelet aggregometry. A lupus inhibitor screen was negative. Paraprotein assessment demonstrated an IgA lambda paraprotein measuring $7 \mathrm{~g} / \mathrm{L}$.

Given her history of bleeding, this patient received four units of fresh frozen plasma prior to the permanent pacemaker insertion but had no intra-operative or post-operative bleeding. She was then lost to follow-up for several years but referred back for assessment after having delayed bleeding following tooth extractions. Repeat coagulation studies were unchanged compared with her studies 7 years prior. In addition, her paraprotein was stable.

The third patient was a 46-year-old male who presented with minor bleeding not requiring intervention related to low risk surgical procedures (minor liposuction, removal of axillary sweat glands) and was noted to have a prolonged APTT of $57 \mathrm{~s}$ that did not correct on mixing (55 s). He was not receiving any antiplatelet or anticoagulant therapy. Factor level testing demonstrated a reduction in levels of all the 
Table 1 Patient results

\begin{tabular}{|c|c|c|c|c|}
\hline \multicolumn{5}{|c|}{ Basic coagulation profile } \\
\hline & Patient 1 & Patient 2 & Patient 3 & Normal range \\
\hline PT & 13 & 15 & 16 & $11-15 \mathrm{~s}$ \\
\hline APTT & 45 & 51 & 57 & $25-35 \mathrm{~s}$ \\
\hline APTT 50/50 mix & 36 & 49 & 55 & \\
\hline Fibrinogen & 4.2 & 3.3 & 2.3 & $2-4 \mathrm{~g} / \mathrm{L}$ \\
\hline Dilution & Factor VIII & Factor IX & Factor XI & Factor XII \\
\hline $1 / 10$ & $71 \%$ & $56 \%$ & $15 \%$ & $75 \%$ \\
\hline $1 / 20$ & $95 \%$ & $76 \%$ & $16 \%$ & $99 \%$ \\
\hline $1 / 40$ & $125 \%$ & $90 \%$ & $20 \%$ & $111 \%$ \\
\hline $1 / 80$ & $134 \%$ & $106 \%$ & $27 \%$ & \\
\hline $1 / 100$ & & & $30 \%$ & \\
\hline $1 / 160$ & & & $42 \%$ & \\
\hline $1 / 200$ & & & $60 \%$ & \\
\hline \multicolumn{5}{|c|}{ Patient 1: Intrinsic factor assays following chemotherapy } \\
\hline Dilution & Factor VIII & Factor IX & Factor XI & Factor XII \\
\hline $1 / 10$ & $112 \%$ & $77 \%$ & $24 \%$ & $94 \%$ \\
\hline $1 / 20$ & $132 \%$ & $85 \%$ & $26 \%$ & $123 \%$ \\
\hline $1 / 40$ & $149 \%$ & $104 \%$ & $32 \%$ & $136 \%$ \\
\hline $1 / 80$ & $164 \%$ & $115 \%$ & $42 \%$ & $131 \%$ \\
\hline \multicolumn{5}{|c|}{ Patient 2: Intrinsic factor assays } \\
\hline Dilution & Factor VIII & Factor IX & Factor XI & Factor XII \\
\hline $1 / 10$ & $3 \%$ & $1 \%$ & $1 \%$ & $4 \%$ \\
\hline $1 / 20$ & & & $1 \%$ & $8 \%$ \\
\hline $1 / 40$ & $13 \%$ & $5 \%$ & & \\
\hline \multicolumn{5}{|c|}{ Patient 3: Intrinsic factor assays } \\
\hline Dilution & Factor VIII & Factor IX & Factor XI & Factor XII \\
\hline $1 / 5$ & $19 \%$ & $22 \%$ & & \\
\hline $1 / 80$ & $87 \%$ & & & \\
\hline \multicolumn{5}{|c|}{ Patient 2: Factor VIII (\%) in inhibitor assay } \\
\hline \multirow[t]{2}{*}{ Incubation dilution } & \multicolumn{4}{|c|}{ Dilution in factor assay } \\
\hline & $1 / 10$ & $1 / 20$ & $1 / 40$ & $1 / 80$ \\
\hline $1 / 5$ & 4 & & & \\
\hline $1 / 40$ & 20 & 28 & 40 & 56 \\
\hline
\end{tabular}

intrinsic factors at $1 / 10$ dilution: Factor VIII $30 \%$, Factor IX $42 \%$, Factor XI 14\% and Factor XII 26\% (Table 1). The factor assays demonstrated increasing concentration with dilution of plasma. Testing for a lupus inhibitor was initially negative, but when repeated, demonstrated a weak lupus type anticoagulant using both tissue thromboplastin inhibition test and Russell's viper venom techniques. The coagulation profile abnormalities were initially attributed to the presence of the weak lupus type inhibitor, although the clinical presentation of bleeding was quite atypical for a lupus inhibitor. PFA-100 testing was normal in this patient.

The patient then underwent rhytidectomy which was complicated by significant, uncontrolled bleeding and fresh frozen plasma was administered. He also had delayed bleeding post-operatively that required further plasma administration. Further work-up then identified an IgG lambda paraprotein measuring $13 \mathrm{~g} / \mathrm{L}$. Bone marrow biopsy demonstrated 5-10\% plasma cells and there was no evidence of end organ damage.
The patient has been followed up for 15 years with no further episodes of bleeding, however has not had any haemostatic challenges. He has had no progression of his plasma cell dyscrasia.

We report three cases of abnormal coagulation results identified in a pre-operative screen that after work-up have been attributed to an underlying MGUS. All three cases had a prolonged APTT that did not correct on mixing and intrinsic factor studies that demonstrated increasing factor levels with dilution, suggestive of an inhibitory effect. Two of the three patients demonstrated a bleeding phenotype.

Abnormal coagulation study results are seen relatively commonly in patients with multiple myeloma, although may not predict bleeding and are often asymptomatic. ${ }^{3-5}$ In one series of patients with multiple myeloma, $37 \%$ had an elevated prothrombin time. ${ }^{4}$ They have also been described in AL amyloidosis cohorts, ${ }^{7}$ however there are limited data regarding their prevalence in MGUS. 
Previously described mechanisms of abnormal coagulation studies in plasma cell dyscrasias include monoclonal protein interference with fibrin polymerisation ${ }^{8}$ and less commonly auto-antibody behaviour targeting specific proteins ${ }^{9,10}$ or heparin-like activity. 5,11,12 AL amyloidosis may also be associated with Factor $\mathrm{X}$ deficiency, attributed to adsorption of Factor $\mathrm{X}$ to the amyloid fibrils. The mechanism of inhibition of multiple intrinsic factors in our cohort is unclear. The difference in bleeding phenotype between the three patients may be explained by the pattern of factor inhibition. The first patient had predominant inhibition of Factor XI and did not demonstrate a bleeding phenotype, while the other two patients, who did have bleeding complications following surgical procedures, had inhibition of multiple factors, most profound in the second patient.

Our case series suggests that patients with an incidental finding of prolonged APTT that does not correct on mixing should be evaluated for the presence of a paraprotein. However, the prolonged APTT may not predict bleeding phenotype. None of our patients have received treatment for their plasma cell dyscrasias or lymphoproliferative disorders thus far, and so it is not possible to evaluate whether their coagulation study abnormalities would improve with reduction in their paraprotein. Prospective evaluation of coagulation profiles in patients with newly diagnosed MGUS could provide epidemiological data regarding the prevalence of abnormal coagulation profiles in these patients.

Conflicts of interest and sources of funding: The authors state that there are no conflicts of interest to disclose.

\section{Georgia McCaughan ${ }^{1}$, Susan Jarvis ${ }^{1}$, Joanne Joseph ${ }^{1,2}$}

${ }^{1}$ Department of Haematology, St Vincent's Hospital, Sydney, NSW, Australia; ${ }^{2}$ Faculty of Medicine, University of New South Wales, Sydney, NSW, Australia

Contact Dr Georgia McCaughan.

E-mail: Georgia.mccaughan@svha.org.au

1. Rajkumar SV, Dimopoulos MA, Palumbo A, et al. International Myeloma Working Group updated criteria for the diagnosis of multiple myeloma. Lancet Oncol 2014; 15: e538-48.

2. Kristinsson SY, Pfeiffer RM, Bjorkholm M, et al. Arterial and venous thrombosis in monoclonal gammopathy of undetermined significance and multiple myeloma: a population-based study. Blood 2010; 115: 4991-8.

3. Perkins HA, MacKenzie MR, Fudenberg HH. Hemostatic defects in dysproteinemias. Blood 1970; 35: 695-707.

4. Kyle RA, Gertz MA, Witzig TE, et al. Review of 1027 patients with newly diagnosed multiple myeloma. Mayo Clin Proc 2003; 78: 21-33.

5. Eby C. Pathogenesis and management of bleeding and thrombosis in plasma cell dyscrasias. Br J Haematol 2009; 145: 151-63.

6. Rodeghiero F, Tosetto A, Abshire T, et al. ISTH/SSC bleeding assessment tool: a standardized questionnaire and a proposal for a new bleeding score for inherited bleeding disorders. J Thromb Haemost 2010; 8: 2063-5.

7. Mumford AD, O’Donnell J, Gillmore JD, Manning RA, Hawkins PN, Laffan M. Bleeding symptoms and coagulation abnormalities in 337 patients with AL-amyloidosis. Br J Haematol 2000; 110: 454-60.

8. Kotlin R, Sobotkova A, Riedel T, et al. Acquired dysfibrinogenemia secondary to multiple myeloma. Acta Haematol 2008; 120: 75-81.

9. Colwell NS, Tollefsen DM, Blinder MA. Identification of a monoclonal thrombin inhibitor associated with multiple myeloma and a severe bleeding disorder. Br J Haematol 1997; 97: 219-26.

10. Wu XY, Yin YF, Teng JL, Zhang LW, Yang CD. IgMk paraprotein from gammopathy patient can bind to cardiolipin and interfere with coagulation assay: a case report. BMC Immunol 2017; 18: 32.
11. Llamas P, Outeirino J, Espinoza J, Santos AB, Roman A, Tomas JF Report of three cases of circulating heparin-like anticoagulants. Am J Hematol 2001; 67: 256-8.

12. Palmer RN, Rick ME, Rick PD, Zeller JA, Gralnick HR. Circulating heparan sulfate anticoagulant in a patient with a fatal bleeding disorder. N Engl J Med 1984; 310: 1696-9.

DOI: https://doi.org/10.1016/j.pathol.2020.11.005

\section{A case report of successful transition from argatroban to warfarin using DOAC-stop in heparin induced thrombocytopenia (HIT)}

\section{Sir,}

Heparin induced thrombocytopenia (HIT) is a serious lifethreatening complication due to exposure to heparin or low molecular weight heparin that necessitates anticoagulation with an alternative agent. A commonly used agent is argatroban, a direct thrombin inhibitor (DTI) which is safe to use in patients with concomitant renal failure. At some point there needs to be a switch from the argatroban infusion to an oral agent such as warfarin if direct oral anticoagulants (DOACs) are contraindicated. The conversion from argatroban to warfarin can be difficult and there are no clear guidelines for this transition. The largest study of argatroban use in HIT, where 418 patients were treated and $63 \%$ were transitioned to warfarin, did not define the transition protocol or guideline used.

There are published suggestions on argatroban to warfarin transition used by different haematology centres. The most common method involves co-administration of argatroban and wafarin for 5 days, cessation of argatroban when the INR $>4$, repeat INR after 4 hours to ensure the warfarin is effective with a target INR of $2-3$ and cessation of argatroban if this has occurred. If this has not occurred, the argatroban is re-commenced and these steps are repeated.

An analysis of outcomes of patients who underwent an argatroban to warfarin transition as mentioned above was carried out by Bartholomew et al. $^{3}$ They identified 111 patients who achieved an INR $>4$ during combined argatroban and warfarin therapy. Adverse outcomes occurred in nine $(8.1 \%)$ patients during co-therapy and $12(10.8 \%)$ patients after argatroban was discontinued, including seven new thrombosis, 12 deaths $(11 / 12$ died due to causes other than thrombosis) and one major bleed. Their conclusion was that in patients receiving argatroban/warfarin co-therapy and an INR $>4$, the risk of thrombosis exceeds the risk of bleeding. These data suggest that the traditional method of ceasing argatroban to check the warfarin induced change in INR in a population of patients already at high risk of thrombosis may not be an ideal method.

We report a case of argatroban to warfarin transition using DOAC-Stop, a commercial product that removes all types of DOACs (dabigatran, apixaban, rivaroxaban and edoxaban) from plasmas. Whilst DOAC-Stop has been used in the laboratory to overcome DOACs to good effect, this is the first described case of using DOAC-Stop to neutralise the effects of argatroban. ${ }^{4,5}$ 